\title{
Erratum
}

\section{Effects of Endurance Exercise Training on The Motor and Non-Motor Features of Parkinson's Disease: A Review}

Guillaume Lamotte, Miriam R. Rafferty, Janey Prodoehl, Wendy M. Kohrt, Cynthia L. Comella, Tanya Simuni and Daniel M. Corcos

[Journal of Parkinson's Disease 5(1), 2015, 21-41, DOI 10.3233/JPD-140425]

On page 30, column 2, line 4, the intensity of HRmax was given as 79.7\%, but this is incorrect. The correct sentence should be:

"Therefore, this interventional study provides only Class IV level of evidence that a moderate to vigorous endurance exercise program (mean HR=69.7\% HRmax) improves motor function in mild to moderate PD [36]." 